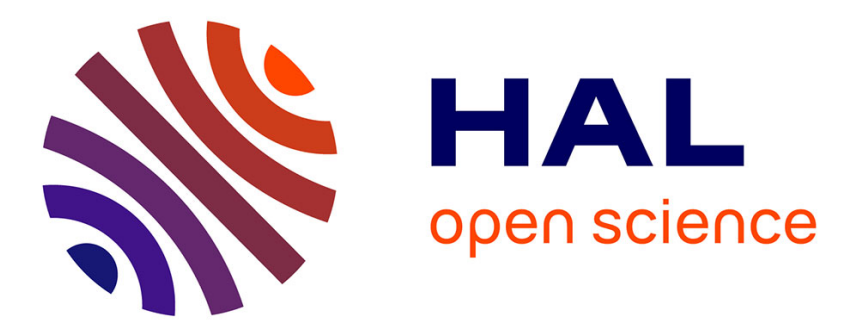

\title{
Airborne ultrasound surface motion camera: application to seismocardiography
}

Pavel Shirkovskiy, Alexandre Laurin, Nathan Jeger-Madiot, Dominique

Chapelle, Mathias Fink, Ros Kiri Ing

\section{- To cite this version:}

Pavel Shirkovskiy, Alexandre Laurin, Nathan Jeger-Madiot, Dominique Chapelle, Mathias Fink, et al.. Airborne ultrasound surface motion camera: application to seismocardiography. Applied Physics Letters, 2018, 112, 10.1063/1.5028348 . hal-01791827

\section{HAL Id: hal-01791827 \\ https://hal.inria.fr/hal-01791827}

Submitted on 14 May 2018

HAL is a multi-disciplinary open access archive for the deposit and dissemination of scientific research documents, whether they are published or not. The documents may come from teaching and research institutions in France or abroad, or from public or private research centers.
L'archive ouverte pluridisciplinaire HAL, est destinée au dépôt et à la diffusion de documents scientifiques de niveau recherche, publiés ou non, émanant des établissements d'enseignement et de recherche français ou étrangers, des laboratoires publics ou privés. 
Title: Airborne ultrasound surface motion camera: application to seismocardiography

Authors: P. Shirkovskiy ${ }^{1}$, A. Laurin ${ }^{2,3}$, N. Jeger-Madiot ${ }^{1}$, D. Chapelle ${ }^{2,3}$, M. Fink ${ }^{1}$ and R.K. Ing ${ }^{1}$

Affiliation:

${ }^{1}$ Institut Langevin, CNRS, ESPCI Paris, PSL Research University, 1 rue Jussieu, 75238

Paris Cedex 05, France

${ }^{2}$ Inria, Université Paris-Saclay, 1 rue Honoré d'Estienne d'Orves, 91120 Palaiseau, France

${ }^{3}$ LMS, Ecole Polytechnique, CNRS, Université Paris-Saclay, 91128 Palaiseau Cedex, France

Abstract: The recent achievements in the accelerometer-based seismocardiography field indicate a strong potential for this technique to address wide variety of clinical needs. Recordings from different locations on the chest can give a more comprehensive observation and interpretation of wave propagation phenomena than a single-point recording, can validate existing modeling assumptions (such as the representation of the sternum as a single solid body), and provide better identifiability for models using richer recordings. Ultimately, the goal is to advance our physiological understanding of the processes to provide useful data to promote cardiovascular health. Accelerometer-based multichannel system is a contact method and laborious for use in practice, also even ultralight accelerometers can cause non-negligible loading effects. We propose a new contactless ultrasound imaging method to measure thoracic and abdominal surface motions, demonstrating that it is adequate for typical seismocardiogram use. The developed method extends non-contact surface-vibrometry to fast $2 \mathrm{D}$ mapping by originally combining multi-element airborne ultrasound arrays, a synthetic aperture implementation and pulsed-waves. Experimental results show the ability of the developed method to obtain 2D seismocardiographic maps of the body surface $30 \times 40 \mathrm{~cm}^{2}$ in dimension, with a temporal sampling rate of several hundred $\mathrm{Hz}$, using ultrasound waves with the central frequency of $40 \mathrm{kHz}$. Our implementation was validated in-vivo on eight healthy human participants. The shape and position of the zone of maximal absolute acceleration and velocity during the cardiac cycle were also observed. This technology could potentially be used to obtain more complete cardio-vascular information than single-source SCG in and out of clinical environments, due to enhanced identifiability provided by distributed measurements, and observation of propagation phenomena.

Keywords: Non-contact ultrasonic imaging, surface motion, non-contact seismocardiography, airborne ultrasound vibrometry, cardio-vascular signals, cardiac mechanics

\section{INTRODUCTION}

Generally Seismocardiogram (SCG) systems have used accelerometers [1] or microwave radars [2] to measure the thoracic surface motion produced by the heart beats. With a few exceptions, these methods have been limited to single-point measurements on the chest surface. While automatic annotation algorithms have been developed [3,4], and published studies demonstrate potential uses for clinical applications [5-9], SCG has so far largely remains confined to scientific laboratories. This is in part due to the lack of physiological and physical understanding of the processes that underlie the phenomena, causing lasting uncertainties as to the exact interpretation of the signal's peaks and valleys, as well as some variability in accelerometer placement. 
A 2D time-map of chest wall movements would provide more complete data, and thus help in the physiological and clinical interpretation of SCG. Although some initial work has been done on array-acquisition of SCG $[10,11]$, physiological interpretation of these data remain either absent, or tenuously linked to individual valve events.

This study's proposed system uses airborne ultrasonic waves to detect movement in a roughly $30 \times 40 \mathrm{~cm}^{2}$ area of interest without direct contact. Some other methods were explored to achieve a similar purpose, namely laser speckle imaging $[12,13]$, scanning laser vibrometry [11, 14] and stereoscopic video [15]. These latter methods were dismissed due to their respective limitations: the lower sensitivity for techniques using video cameras [16], the necessity to synchronize heart beat ECG with laser vibrometer in the sequential scanning mode, the need to have a good reflective surface for all optical based systems. Furthermore, due to implementation difficulties all these techniques have usually been limited to 30 acquisition channels on $\sim 25 \times 25$ $\mathrm{cm}^{2}$ area $[10,11]$.

Airborne ultrasonic waves of low power in measurements have the advantage of being lowrisk, and having lower cost of implementation. The first patents for non-contact measurement of body movement with ultrasound were filed in the 70s [17, 18]. Later studies have described and validated ultrasound-based systems for various monitoring applications [19, 20]. The acoustical methods required for 2D surface vibrometry with time- and velocity-resolutions adequate for SCG have only recently become feasible [21].

Our goal in this study was to demonstrate a new non-contact method for non-plane surface vibrometry. It consists in an airborne ultrasound surface motion camera (AUSMC) that is able to achieve multi-channel SCG data measurements on the human thorax and abdomen.

A secondary goal was to validate the capability of the AUSMC system to perform 2D SCG measurements and to perform qualitative examination of the $2 \mathrm{D}$ velocity map under cardiac forces to develop hypotheses of the thoracic and abdominal systems as a whole. The underlying aim was to explore the developed AUSMC system to complement existing techniques for performing meaningful medical measurements, and to understand the mechanical principles behind cardiothoracic movements.

\section{MATERIAL AND METHODS}

The AUSMC system is composed of nine emission panels arranged in a square matrix (Fig.1a) and one reception panel positioned at the same location as the central emission panel. Each emission panel is composed of 32 piezoelectric transducers (Murata MA40S4S) uniformly distributed over an area of $24 \times 24 \mathrm{~cm}^{2}$ dimension and operating around the central frequency of $40 \mathrm{kHz}$. The central panel combines both the emitting and receiving elements on the same plane. The reception panel is composed of $16 \times 16$ square array of receivers (Knowles microphone FG23629 equipped with $40 \mathrm{~dB}$ electronic amplifier). The horizontal and vertical pitch of microphones is $16 \mathrm{~mm}$ i.e. nearly twice the wavelength in the air. A camera is included in the experimental setup to overlay a visual representation of the participant to the acoustical images.

This configuration addresses several imaging challenges:

1. The physical separation between emitter and receiver arrays enables simultaneous emission and reception. In this configuration the round-trip time is no longer a constraint, and operation with higher frequency rate of ultrasound illumination is allowed.

2. Multiple panels are used to increase the surface of the emission aperture and to ensure an adequate imaging surface, comparable with the size of receiving aperture. At $40 \mathrm{kHz}$, the 
wavelength in the air is roughly equal to $8.6 \mathrm{~mm}$. The roughness of the skin is less than $300 \mu \mathrm{m}$. Because of an acoustic impedance four order of magnitude higher than that of the air, the skin acts as a specular surface, unlike the diffusion regime where the acoustic wave of illumination would be randomly backscattered. To ensure that all the imaged surface reflect acoustic waves toward the whole reception array, an emission aperture nine times larger than receiving is used. The whole system works in synthetic aperture mode [22] where after each complete step of illumination the whole acoustic image is computed from the impulse responses between all the emitting transducers and receiving microphones. However, to get a high framerate in the synthetic aperture mode the number of impulse responses to measure must be maintained low. In this context all transducers at the same position on each of the nine emission panels are electrically connected together (Fig.1b). Finally, the number of independent emission channels are reduced from 288 to only 32. Each independent channel drives nine transducers located on nine distinct emission panels. Despite the fact that nine transducers (each on its own panel) radiate simultaneously and because of long distance between them and specular reflection, an acoustic wave generated mainly from one single transducer is reflected toward the reception aperture for a given point of the observation surface. Acoustic waves from the eight other transducers are reflected toward the outside region.

This setup is valid if the measurement surface is planar and parallel to the whole aperture. It is less efficient when the measurement surface is curved. However, as the experimental results show, the AUSMC system is still usable on the torso on individuals even if some areas are not sufficiently illuminated.

Emission channels are driven by the 32 electrical outputs of a programmable D/A acquisition card D-TACQ ACQ1001. Each channel is connected to 9 transducers and the voltage amplitude should reach $20 \mathrm{~V}$ pk-pk. The emission waveform is a linearly modulated frequency signal that ranges from $35 \mathrm{kHz}$ to $45 \mathrm{kHz}$. Its time duration is $400 \mu \mathrm{s}$. The emission channel is excited in a fixed order. Successive emissions are delayed by $200 \mu$ s. The emission sequence enclosing the excitation of the 32 emission channels lasts $6.4 \mathrm{~ms}$. It is repeated continuously during $12 \mathrm{~s}$. The framerate is $1 / 6.4 \mathrm{~ms}$ and then equal to $157 \mathrm{~Hz}$.

The receiver array is connected to a 256-channels A/D acquisition card D-TACQ ACQ196 (16 bits, $320 \mathrm{kSamples} / \mathrm{s}$ ) and reflected wavefronts are recorded continuously during the entire emission time up to $12 \mathrm{~s}$.

\section{$\underline{\text { SIGNAL PROCESSING }}$}

The AUSMC system works in the synthetic aperture mode. During one illumination step all the 32 emission channels emit successively to acquire the impulse responses from one channel to the entire 256 microphones. Thereafter a beamforming is both processed in transmission and reception modes. For the transmission mode only one of nine transducers is taken into account. The choice depends on the point of the observation surface and reflection law assuming the observation surface to be planar. The beamforming in transmission mode is achieved by linearly combining the signals from the 32 output channels. The beamforming in reception mode is realized thereafter and results in an acoustic signal for each point of observation of the surface. Generally, the observed surface is decomposed into $60 \times 80$ pixels i.e. $5 \mathrm{~mm}$ pitch for both horizontal and vertical directions. Each illumination step lasts $6.4 \mathrm{~ms}$. The following illumination step leads to the same computation process as described below. The surface motion for each pixel is then determined by cross correlating the acoustic signals computed for two successive illumination steps. The out of plane surface displacement amplitude between the two illumination steps is equal 
to the half of the product between the time delay between the two acoustic signals and air acoustic velocity. The frame rate of the surface motion video is equal to the inverse of the illumination step duration and is equal to $157 \mathrm{~Hz}$. The surface motion computed corresponds to the differential out of plane displacement amplitude of the surface and therefore corresponds to the out of plane velocity of the surface. Out of plane acceleration is obtained by time differentiation. The important advantage of this method is its ability to realize both beamforming in transmission and reception modes from the impulse responses acquired during each illumination step. Finally, it allows to get a high framerate with a good focusing dynamic - i.e. focusing lobe level vs side lobe level - and a reasonable spatial resolution.

\section{$\underline{\text { SEISMOCARDIOGRAMS }}$}

Investigation of the AUSMC device for SCG monitoring was performed on eight healthy male subjects (23-35 years old). The study was approved by the appropriate legal and ethical authority (Comité de Protection des Personnes (CPP) de Sud-Méditerranée II en date du $12 / 05 / 2017$ ). The participants are sitting in front of the measurement system - at a $720 \mathrm{~mm}$ distance - with blocked respiration. Acquisition time was 12 seconds. AUSMC, accelerometer-based and laser vibrometer (LV) measurements for some points are performed concurrently for comparison purposes.

\section{$\underline{\text { RESULTS }}$}

We reported here a new method for surface motion monitoring, and tested its capability to acquire $2 \mathrm{D}$ vibration maps on human torso. The developed device provided in non-contact mode surface acceleration, velocity or displacement maps over time of the $30 \times 40 \mathrm{~cm} 2$ area.

Accelerometer-based, LV and AUSMC SCG signals received from the same places are bandpass filtered at $15-60 \mathrm{~Hz}$ and compared. Both signals from accelerometer and laser vibrometer and signal from the AUSMC device at the same measurement point match well, especially when we consider the temporal waveform (Fig. 4 Top and Middle).

Before mapping the body surface motion, we first characterized the spatial resolution and sensitivity of our system. The spatial resolution was determined using a small disc of $7 \mathrm{~mm}$ diameter acting as a point like reflector and located at $720 \mathrm{~mm}$ distance. The focusing diameter at $-6 \mathrm{~dB}$ is found to be equal to $30 \mathrm{~mm}$ (Fig. 2). This value is equal to 3.5 times the aerial wavelength at $40 \mathrm{kHz}$ for a focal length to aperture width ratio equal to 3. The sensitivity of the AUSMC system is limited by the digital noise of the calculated out of plane velocity images. This noise is of normal distribution with a standard deviation equal to $95 \mu \mathrm{m} / \mathrm{s}$ (Fig. 3).

We observed the shape and position of the zone of maximal absolute velocity and acceleration during cardiac cycle (Fig. 5). We also observed a downward-propagating wave on the abdomen. We estimated its velocity by computing the slope of the slanted sections of each systolic wave train on the central-line velocity maps (Fig. 4 Bottom). The mean velocity for the 8 participants was $2.6 \pm 0.4 \mathrm{~m} / \mathrm{s}$.

\section{$\underline{\text { DISCUSSION }}$}

The study presents preliminary results that support the capability of the AUSMC system to perform SCG measurements in non-contact mode. It is clear, though, that the AUSMC system can 
potentially go beyond the measurement of SCG timings and amplitudes, by allowing the observation of the motion of the entire thorax and abdomen during heart beats. This multi-channel system can give a greater understanding of the relationship between the biological signals in SCG and the physiological phenomenon creating them.

The slanted sections of each systolic period, a wave propagation phenomenon of $\sim 2.6 \pm 0.4$ $\mathrm{m} / \mathrm{s}$ that is observed in all participants on the abdomen, is much too slow to be the pulse wave of the descending aorta, which travels at $\sim 10 \mathrm{~m} / \mathrm{s}$ in a healthy normotensive human [23]. We have been unable to identify an existing phenomenon observed by clinicians propagating at similar speeds. As such, it presents an intriguing, completely new observation that may have been made possible only with the development of the techniques described in this paper. This wave could potentially be caused by the heart pulling and pushing on the diaphragm, which then propagates in the general viscera, and the velocity could then be related to fat distribution, muscle tone, or other physiologically relevant variables. Studies examining this specific observation have been designed and started by our team.

Results are consistent with the hypothesis that the sternum moves as a solid object under heart forces. These observations are also compatible with the idea that the thoracic cage reacts to cardiac forces as a rather stiff structure, whose movements could be adequately represented with some relatively small set of vibration modes. This is of much value for model validation purposes. Furthermore, distributed measurements enhance identifiability, and therefore we expect more comprehensive and accurate characterization of model properties.

Obtained 2D maps can help for development of new version of the AUSMC system more adapted to the SCG needs and for creation and optimization multisensory accelerometer systems, for example for wearable SCG.

\section{CONCLUSION}

A new method was developed for non-contact surface-vibrometry by combining multielement airborne ultrasound arrays, a synthetic aperture implementation and pulsed-waves. Experimental results show the ability of the developed method to obtain 2D surface motion maps with frame rate of several hundred $\mathrm{Hz}$, however, the principle of operation makes it possible to achieve $1 \mathrm{kHz}$ of frame rates using ultrasound waves with the central frequency of $40 \mathrm{kHz}$.

As a valid method for measuring SCG timings, the AUSMC system could provide a quicker, less invasive alternative to existing accelerometer-based techniques. In a routine visit to the doctor, for example, a device embedded in the wall could perform measurements while the patient is standing on the weighing scale, minimizing effort and time. Also seismocardiographic data collection from multiple locations through multiple channels may help increase the reliability of the method. The AUSMC system has the potential to unlock a new dimension in the study of SCG, although more work is required to establish it firmly. Thus the ultrasound non-contact multichannel SCG measurement system can be a promising technology for 2D cardiac mechanical signal recording, and, in conjunction with ECG, which records electrical cardiac signals, may become an important diagnostic tool in the clinical diagnosis of cardiac diseases, such as valvular heart diseases, heart failure and ventricular dyssynchrony.

\section{$\underline{\text { ACKNOWLEDGEMENTS }}$}

This work was supported by Chaire Safran - Fonds ESPCI Georges Charpak. 


\section{$\underline{\text { REFERENCES }}$}

${ }^{1}$ O. Inan, P. Migeotte, K. Park, M. Etemadi, K. Tavakolian, R. Casanella, J. Zanetti, J. Tank, I. Funtova, G. Prisk and M. Di Rienzo, "Ballistocardiography and Seismocardiography: A Review of Recent Advances", IEEE journal of biomedical and health informatics, vol. 19, no. 4, 14141427 (2015).

2 K. Tavakolian, F.M. Zadeh, Y. Chuo,T. Siu, A. Vaseghi, B. Kaminska, "Radar mechanocardiography: A novel analysis of the mechanical behavior of the heart", Conf Proc IEEE Eng Med Biol Soc. 2008; 4863-6 (2008). doi: 10.1109/IEMBS.2008.4650303.

3 A. Laurin, F. Khosrow, A. Blaber, K. Tavakolian, "Accurate and consistent automatic seismocardiogram annotation without concurrent ECG", Computing in Cardiology Conference (CinC), 2015. DOI: 10.1109/CIC.2015.7408577.

${ }^{4}$ F. Khosrow-khavar, K. Tavakolian, A.P. Blaber, J.M. Zanetti, R. Fazel-Rezai, C. Menon, "Automatic Annotation of Seismocardiogram with High Frequency Precordial Accelerations", IEEE J Biomed Health Inform, 19(4), pp. 1428-34 (2015).

${ }^{5}$ D.M. Salerno, J.M. Zanetti, "Seismocardiography for monitoring changes in left ventricular function during ischemia", Chest., 100(4), pp. 991-3 (1991).

${ }^{6}$ D.M. Salerno, J.M. Zanetti, L.A. Green, M.R. Mooney, J.D. Madison, R.A. Van Tassel, "Seismocardiographic changes associated with obstruction of coronary blood flow during balloon angioplasty", The American journal of cardiology, vol. 68, no. 2, pp. 2017 (1991).

${ }^{7}$ D.M. Salerno, J.M. Zanetti, R. Crow, "Seismocardiography for detection of coronary artery disease", Am. J. Noninvasive Cardiol., 6, pp. 321-330 (1992).

${ }^{8}$ K. Tavakolian, G. Houlton, G. A. Durmont et al., "Precordial vibrations provide noninvasive detection of early-stage hemorrhage", J. Shock, vol. 41, pp. 91-96, (2014).

${ }^{9}$ A. Laurin, A. Blaber, K. Tavakolian, "Seismocardiograms return Valid Heart Rate Variability Indices", Computing in Cardiology Conference (CinC), 2013.

${ }^{10}$ M. Cozic, L.G. Durand, R. Guardo, "Development of a cardiac acoustic mapping system”, Med Biol Eng Comput, 36(4), 431-437 (1998).

${ }^{11}$ K. Munck, B.D. Hansen, N. Jacobsen, L.P. Pilgaard, S. Schmidt, K. Sørensen, J.J. Struijk, "Body Surface Mapping of the Mechanical Cardiac Activity", Computing in Cardiology, vol. 43 ISSN: 2325-887X DOI:10.22489/CinC.2016.193-348 (2016).

12 G. Ramachandran and M. Singh, "Cardiac Functional Analysis by Laser Speckle Interferometry", vol. 11, 233-239 (1989).

${ }^{13}$ M. Singh and G. Ramachandran, "Reconstruction of sequential cardiac in-plane displacement patterns on the chest wall by laser speckle interferometry", IEEE Trans. Biomed. Eng., vol. 38, no. 5, 483-9 (1991).

${ }^{14}$ H. Tabatabai, D. E. Oliver, J.W. Rohrbaugh, C. Papadopoulos, "Novel Applications of Laser Doppler Vibration Measurements to Medical Imaging", Sens Imaging 14, pp. 13-28 (2013). 
15 M.-Z. Poh, D.J. McDuff, and R.W. Picard, "Non-contact, automated cardiac pulse measurements using video imaging and blind source separation”, Opt. Express, vol. 18, no. 10, 10762-74 (2010)

${ }^{16}$ U Wijenayake, S.Y. Park, "Real-Time external respiratory motion measuring technique using an RGB-D camera and principal component analysis", Sensors (Basel), 17(8), E1840 (2017). doi: $10.3390 / \mathrm{s} 17081840$.

${ }^{17}$ H. Karsh, Motion monitor. 1978 Oct; 24 U.S. Patent 4,122,427.

${ }_{18}$ R.B. Northrup. Ultrasonic respiration/convulsion monitoring apparatus and method for its use. 1980 Apr; 15 U.S. Patent 4,197,856.

${ }^{19}$ S.D. Min, J.K. Kim, H.S. Shin, Y.H. Yun, C.K. Lee, and M. Lee, "Noncontact respiration rate measurement system using an ultrasonic proximity sensor", Sensors Journal, IEEE, vol. 10, no. 11, 1732-1739 (2010).

${ }^{20}$ N. Jeger-Madiot, J. Gateau, M. Fink, R.K. Ing, "Non-contact and through-clothing measurement of the heart rate using ultrasound vibrocardiography", Medical Engineering and Physics (2017), https://doi.org/10.1016/j.medengphy.2017.09.003.

${ }^{21}$ N. Jeger, M. Fink, R.K. Ing, “3D airborne ultrasound vibrometer for the detection of skin surface heterogeneities", IEEE International Ultrasonics Symposium, Tours (2016).

22 J.A. Jensen, S.I. Nikolov, K.L. Gammelmark, and M.H. Pedersen, "Synthetic aperture ultrasound imaging", Ultrasonics 44 Suppl 1, e5-e15 (2006).

${ }^{23}$ G. Mancia, G. Backer, A. Dominiczak, R. Cifkova, R. Fagard, G. Germano, G. Grassi, A. M. Heagerty, S. E. Kjeldsen, S. Laurent, K. Narkiewicz, L. Ruilope, A. Rynkiewicz, R. E. Schmieder, H. Struijker Boudier, A. Zanchetti, "2007 Guidelines for the management of arterial hypertension: The Task Force for the Management of Arterial Hypertension of the European Society of Hypertension (ESH) and of the European Society of Cardiology (ESC)", European heart journal 28.12, 1462-1536 (2007). 


\section{Captions for Figures}

FIGURE 1. a) Experimental setup. Data acquisition is performed on sitting participants. b) Schematic top view of the emission/reception panel. Each transmission channel among 32 is used to drive 9 transducers distributed on nine separate sub-panels. The diagram shows that only one transducer is effective, the reflected acoustic waves from the other eight are not correctly directed to the central panel containing the receivers.

FIGURE 2. Acoustic image of a small disc of $7 \mathrm{~mm}$ diameter realized with the AUSMC system.

FIGURE 3. Experimental noise density function (blue bar) and normal distribution fitting function (red curve) with a standard deviation equal to $95 \mu \mathrm{m} / \mathrm{s}$.

FIGURE 4. Accelerometer and vibrometer signals compared to the AUSMC signal sampled at points of placement of the sensors (top and middle figures). Mean systolic time: $0.26 \pm 0.03 \mathrm{~s}$, mean diastolic time $0.60 \pm 0.14 \mathrm{~s}$. B-SCAN along the central line passing through the sternum obtained from the signals of the AUSMC system (bottom figure). Velocity is counted positively when pointing outward of the thorax. The velocity of the downward-propagating surface wave on the abdomen is computed from the slope of the slanted regions.

FIGURE 5. Body surface motion mapping of the mechanical cardiac activity. Top left image: picture of test subject with highlighted region of interest. Other images: pictures of out of plane surface motion velocity. Note: propagation of surface waves is observed on the body surface during the systole and diastole phases of cardiac cycle. The color scale represents the value of the out of plane surface motion velocity. MC - mitral valve closure, AC - aortic valve closure. 
0

20

60

80
$0 \quad 20$
40
60
80

$-6$

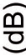

$\frac{1}{\frac{1}{2}}$ 


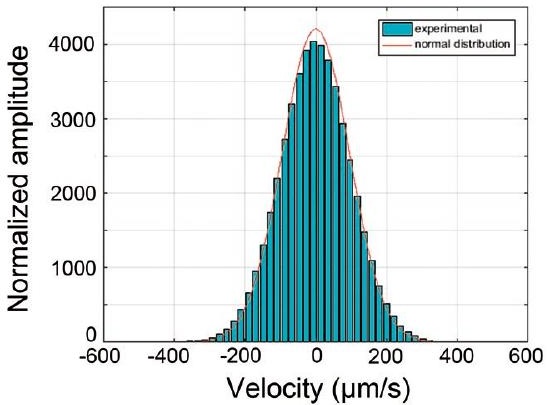


Acceleration $\left(\mathrm{m} / \mathrm{s}^{2}\right)$
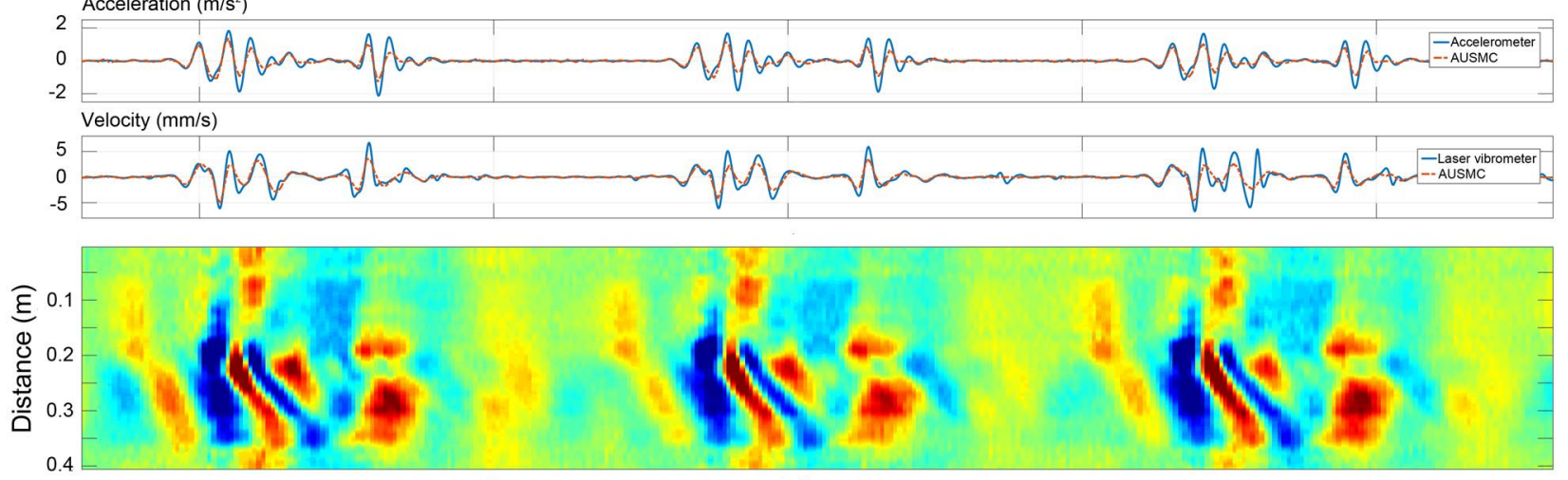

Time (s)

$\begin{array}{cccccc}-10 & -8 & -6 & -4 & -2 & 0 \\ & & & & \text { Velocity }(\mathrm{mm} / \mathrm{s})\end{array}$



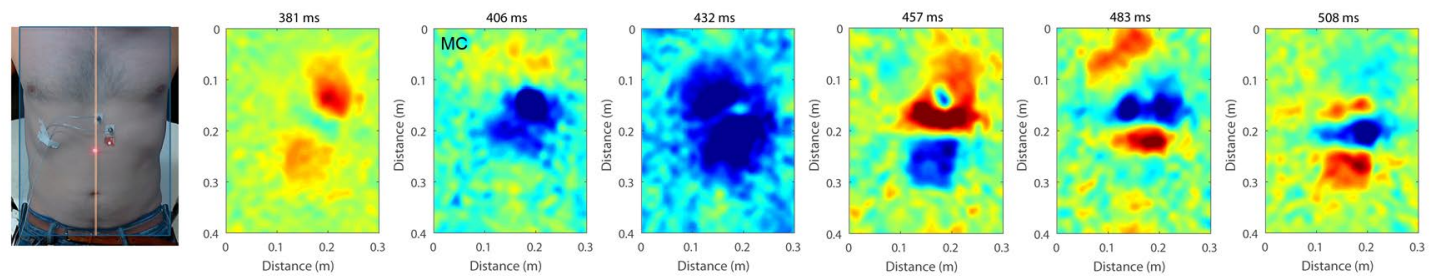

$533 \mathrm{~ms}$
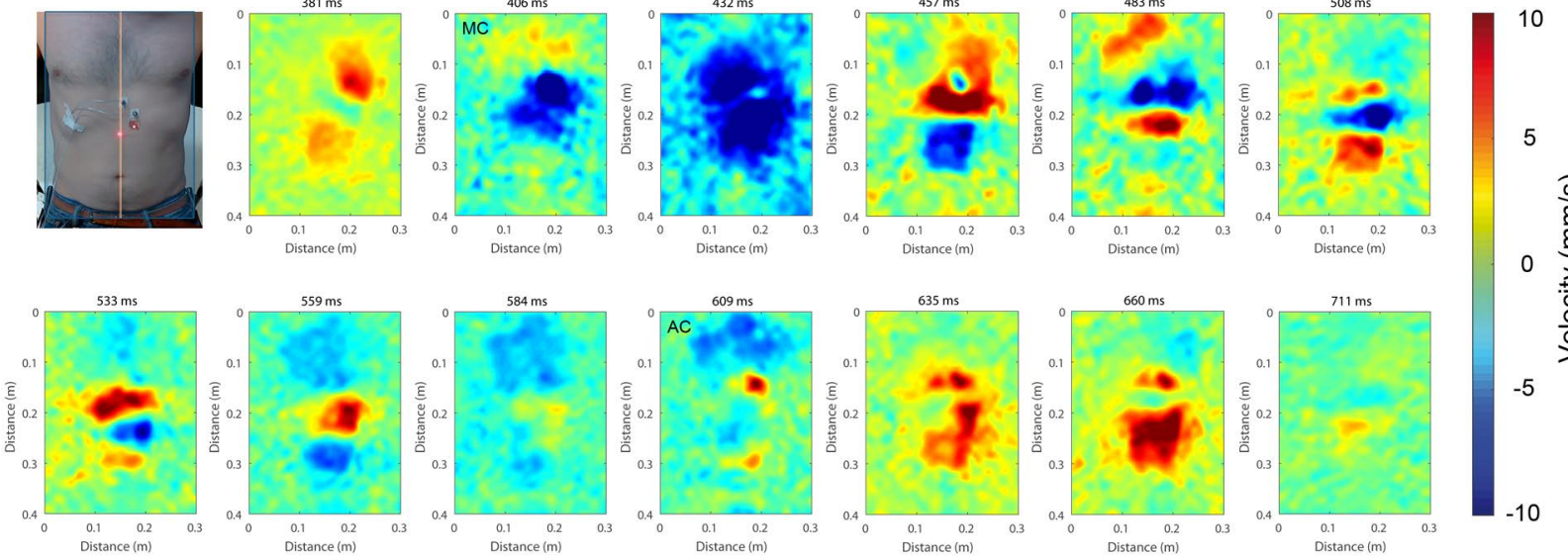